\title{
TRANSILLUMINATION IN THE DIAGNOSIS OF SINUS DISEASE.*
}

\author{
BY CHAS. H. KNIGHT, M.D., NEW YORK.
}

The purpose of this brief paper is to assist in establishing a just estimate of transillumination as a diagnostic test.

No reference will be made to the history or literature of the subject. It is merely a record of personal experience based on a study of more than two hundred cases examined during the last six months. The examinations were made without reference to pathological conditions, although, of course, nost of the subjects had more or less derangement of the upper air passages.

The symptoms of sinus disease are often so obscure that we may be forced to resort to any measure that may facilitate cliagnosis, even though under certain circumstances it may be misleading. Some of the prevailing scepticism as to the value of the light test results from a study of the uncovered skull. For clinical purposes it is obvious that conclusions thus reached may be fallacious, since a relatively small antrum, for example, may have a thin, translucent lining membrane, while on the other hand a capacious antrum may have a dense, impenetrable wall. The transmission of light may be obstructed not only by a thickened mucous membrane, but by various anomalies in the bones constituting the walls of the antrum. Among the latter may be mentioned bony septa subdividing the cavity, exostoses projecting into it, or unusual thickening or distortion of the bony wall. Deficient resorption of the maxillary bones would give a contracted antrum and proportionate diminution of light, while excessive resorption would increase the dimensions of the antrum and give a more intense light. The latter result is said to be associated also with those rare conditions clesignated mucocele and cyst of the antrum. I have had but one opportunity to examine a case of cyst, and in that the light was decidedly brighter on the affected side owing to expansion of the cavity and extreme thinning of its anterior wall. In this case before the light test was employed it was the opinion of several that the antrum was occupied by a solid tumor.

In making these investigations three points were noted: First, the appearance of the pupils; second, perception of the flash of light on \footnotetext{
25, 1898.

*Read before Mreeting of Laryngological Section, New York Academy of Medicine, May
} 
the part of the patient with his eyes closed, and third, the extent of the light aren in the antral region. The first is not important and is. influenced more or less by the thickness of the orbital plate. In the majority of cases the pupils appear brightly illuminated. In observing the second point allowance must be'made for the stupidity or imagination of the patient, and it also is not of much moment. The third point, as the one of chief interest, will be the only one here referred to. The data to be given presently will relate exclusively to the maxillary sinus. Incidentally I may mention that variations in. the dimensions of the frontal sinuses appeared to be more extreme and in a small number of cases a median septum could be distinctly demonstrated.

Four (4) of the cases on my list were unquestionably empjema and gave marked and characteristic results on application of the electric lamp. Two of these were treated by perforation through the alveolus and all have exhibited increase of translucency as improvement has progressed under treatment.

Five (5) cases with suspicious histories showed incquality in light area, and in three of these the symptoms pointed unmistakably to old suppuration of the antrum which had never been recognized.

In eighteen (IS) cases there was more or less difference between the two sides of the face, but in none of these were there any symp-. toms whatever of any form of antral disease.

In two (2) cases both antra were in absolute shadow. Both of those patients were men with very high-arched palates and very flat. faces. One had pronounced hypertrophic rhinitis and the other at large collection of nasal polypi. The conditions were relieved without affecting the opacity of the antra or disclosing pus within them.

In one hundred and seventy-one (I I I) cases no difference in the light area of the two sides could be detected. One of these cases. while under observation had an attack of acute inflammation of the antrum which presented all the classical symptoms and responcled very graphically to the light test.

How should these figures be interpreted? It seems to me that with ordinary care the proportion of cases in which the light may prove delusive is extremely small. An antrum filled with pus must be opaque. Almost the only chance for error is in the case of an asymmetrical skull, of which the larger antrum contains a small quantity of pus. In the absence of subjective symptoms a dark antrum does not authorize a diagnosis of empyema. The evidence furnished by transillumination, therefore, must be regarded as cor- 
roborative rather than by itself conclusive. It may not be infallible, but it surely has its place in adding to the certainty of dingnosis.

In conclusion, I wish to protest against explorative puncture of the antrum as a diagnostic measure. In my opinion it is a dangerous procedure. For instance, in a recent suspected case punctures on three consecutive days disclosed pus on the third trial. To my mind such a result is by no means surprising. If pus was present on the first attempt the experiment was a failure; if the exploring trocar was the source of infection, not an unreasonable supposition, it was worse than useless.

With equal emphasis I would protest against the adoption of the usual methods of surgical interference in all cases of empyema of the antrum. In certain quarters it is the fashion to plunge into every antrum supposed to contain pus. An opening is made through the alveolus, the antral cavity is irrignted, then packed with gauze, or a drainage tube is inserted. In many cases this is wholly unnecessary.

The principle of treatment I would advocate is as follows: In all cáses of pus in the maxillary sinus in which a diseased tooth is not an etiological factor, the proper course to pursue is to cut away all overgrowth of tissue in the region of the ostium maxillare in order to restore normal drainage through the anatomical outlet of the cavity. It is not clificult to wash out and medicate the antrum by this way and in a fair proportion of cases a cure may be thus obtained in a relatively short time with comparatively slight discomfort to the patient. Should improvement fail to follow in the course of a few weeks, we must conclude that the mucous lining of the sinus has undergone degeneration which necessitates curetting of its walls through an artificial opening.

I47 West Fifty-seventh Street. .

\section{Adenoids in the First Year of Childhood-H. Cuvillaler, Paris-} Am. MY't. Surg. Bulletin, Vol. xii, No. 9.

In his experience the author has met with sixty-four cases of adenoids in children under one year of age. Laryngo-tracheitis, laryngismus stridulus, spasm of the glottis and emphysema have. been observed as sequelæ.

This observer resorts to the medicinal treatment in young subjects, employing instillations of menthol in oil; $1: 60$; or resorcin, I to 50 or 25 .

LEDERMAN. 